\title{
Fetal thymus in the middle and late trimesters: Morphometry and development using post-mortem 3.0T MRI
}

\author{
LEILEI YUAN $^{1,2}$, JINFENG CAO $^{3}$, ZHAOHUA WANG $^{2}$, LITAO ZHANG $^{2}$, XIA WANG $^{2}$, \\ YONG WU ${ }^{4}$, JINYE DONG ${ }^{4}$, HUIHUI XIE ${ }^{4}$ and XIANGTAO LIN ${ }^{1,5}$ \\ ${ }^{1}$ Shandong University School of Medicine, Jinan, Shandong 250012; ${ }^{2}$ Department of Radiology, \\ Taian Central Hospital, Taian, Shandong $271000 ;{ }^{3}$ Central Hospital of Zibo, Zibo, Shandong 255020; \\ ${ }^{4}$ Department of MR, Shandong Medical Imaging Research Institute, Jinan, Shandong 250021; \\ ${ }^{5}$ Shandong Provincial Hospital Affiliated to Shandong University, Jinan, Shandong 250012, P.R. China
}

Received May 10, 2018; Accepted January 30, 2020

\section{DOI: $10.3892 / e t m .2020 .9172$}

\begin{abstract}
The present study aimed to investigate the anatomical microstructure, features and signals of the fetal thymus by 3.0T FS-T2 weighted turbo spin echo sequences, which could provide imaging evidence for the evaluation of early-stage development of fetal thymus. In addition, the T2-weighted three-dimensional (3D) sequences and the 3D processing may contribute to the establishment of reference ranges for the fetal thymus. A total of 64 specimens obtained from the fetuses of 16-39 weeks of gestational age (GA) were scanned by 3.0T MRI. Morphological changes and quantitative measurements of the fetal thymus were assessed, including the anteroposterior diameter, width, height, surface area and volume. The shape of fetal thymus varied and the majority were $\mathrm{X}$-shaped, with a narrow top and wide bottom. Morphology measurements demonstrated gradual growth with increasing GA. There were high linear correlations between width, height, surface area and volume and GA. No significant differences were observed between the sexes. Post-mortem 3.0T MRI clearly demonstrated changes in external contours and internal structure with GA. The images and data obtained reflect normal development of the fetal thymus and enrich the imaging data of fetal morphometry.
\end{abstract}

Correspondence to: Dr Xiangtao Lin, Shandong University School of Medicine, 44 Wenhuaxi Road, Jinan, Shandong 250012, P.R. China

E-mail: linxt@sdu.edu.cn

Abbreviations: GA, gestational age; US, ultrasonography; 3D, three-dimensional; MRI, magnetic resonance imaging; TSE, turbo spin echo; TS, thymus; TV, volume of the thymus; TH, thymus height; TW, thymus width

Key words: normal fetal thymus, morphological measurements, prenatal diagnosis, post-mortem 3.0T MRI

\section{Introduction}

The thymus is relatively large at birth and is important for fetal growth and immunity. Previous studies indicate that thymus is a mediator of the associations between fetal undernutrition and atopic and autoimmune disease in infancy and adulthood $(1,2)$. Therefore, quantitative analysis of the thymus is important during fetal development.

Ultrasonography (US) is considered the first choice for monitoring fetal growth. Measurement of fetal thymus contributes to our understanding on the function of the immune system in the fetus. However, in cases of maternal obesity or oligohydramnios with an unfavorable plane of view, US is not the best for visualizing the fetal thymus (3). In addition, the thymus is located at a position behind the upper portion of the sternum, which cannot be clearly displayed by US. According to previous studies (4-9), the proper antenatal US visualization of thymus is dependent on normal maternal body habitus and amniotic fluid volume. Currently, three-dimensional (3D) US presenting more data in an accurate manner has been used in clinical practice, but its extensive application is hampered due to its disadvantages including motion artifacts and fetal irritability. There are also disputes in the validity of US, particularly the reproducibility and accuracy (3).

Currently, fetal magnetic resonance imaging (MRI) has been widely accepted as a complementary modality to US in prenatal diagnosis $(10,11)$ However, it is still a challenge to identify abnormalities, especially in the middle trimester when the fetus is comparatively small and pulse artifacts may present. Furthermore, in the later stages of fetal development, the thymus cannot be easily observed due to changes of fetal position and overlapping of limbs (10). Thus far, extensive studies have been carried out on the development of the fetus using post-mortem MRI (12-14). The major advantages of this method are excellent resolution and stable image quality as it is not restricted in its scanning field and sequences $(15,16)$. In brief, the anatomical structure and precise scalar dimensions of the fetal thymus can be clearly displayed in the MRI.

The present study investigated the anatomical microstructure, features and signals of the fetal thymus by 3.0T FS-T2 weighted turbo spin echo (TSE) sequences. It provided 
imaging evidence for the evaluation of early-stage development of fetal thymus. In addition, the T2-weighted 3D sequences and the three-dimensional processing may contribute to the establishment of reference ranges for the fetal thymus.

\section{Materials and methods}

Specimen selection. The present study was carried out with approval of the Ethical Committee of School of Medicine, Shandong University (approval no. 2012033). Written informed consent was obtained from the guardian(s) of each patient. Clinical information was collected from maternal and neonatal medical records by one investigator blinded to the prenatal findings.

A total of 78 fetal specimens, 16-39 weeks' gestational age (GA), were collected from Shandong Provincial Hospital. The specimens were obtained from medically indicated abortions, spontaneous abortions, fetal deaths or stillbirths, as well as premature deaths. The inclusion criteria were: i) maternal pregnancy record demonstrated an absence of fetal chromosomal abnormality, stressful uterine conditions, genetic disease or diabetes; and, ii) US findings of the fetus during pregnancy and the findings of the post-mortem MRI examination of the specimen indicated no anatomical abnormality. The exclusion criteria were: Multiple pregnancies, maternal pregnancy records showing a documented fetal chromosomal abnormality, presence of stressful intrauterine conditions, a family history of maternal genetic disease or a history of inflammation, sepsis or stressor (16-19).

A total of 64 specimens met the inclusion criteria. As the femur length is more accurate for the determination of the GA than biparietal diameter, head circumference or foot length (20), the femur length of the fetuses was measured using MRI. The GA was obtained according to the morphometric criteria proposed by Guihard-Costa et al (14).

3.OT MR scanning. Post-mortem MRI was performed within $48 \mathrm{~h}$ of mortality. Fetal specimens were imaged with T2-weighted 3D sequences and FS-T2 weighted TSE sequences, using a Siemens Skyra 3.0T system (Siemens Healthineers). A head and neck joint coil was used for the scanning of the thymus. For the T2-weighted 3D sequences, the repetition time was $13.95 \mathrm{msec}$, echo time was $5.2 \mathrm{msec}$, the matrix was $512 \times 512$, voxel size was $0.4 \times 0.4 \times 0.4 \mathrm{~mm}$ and the number of excitations was 2. For the FS-T2-weighted TSE sequences, the repetition time was $6,160 \mathrm{msec}$, echo time was $60 \mathrm{msec}$, the matrix was $640 \times 446$, the voxel size was $0.3 \times 0.3 \times 1.5 \mathrm{~mm}$ and the number of excitations was 4 . The scanning time was approximately $20 \mathrm{~min}$. The field of view and the distance factor were adjusted according to the circumference of the chest to produce a signal-to-noise ratio of $<1.0$.

$3 D$ reconstruction. The $3 \mathrm{D}$ sequences of the thymus were measured using Amira 5.4 software (Thermo Fisher Scientific, Inc.). Initially, the T2-weighted 3D sequence images were aligned using Amira 5.4 software. Subsequently, the silhouette of the fetal thymus was artificial marked with purple lines on each image in the transverse, sagittal and coronal planes (Fig. 1A-C). After tracing, the 3D software system automatically filled the area with red color for correction (Fig. 1D-F). Later, the 3D visualization was built automatically. After the model had been obtained, the anteroposterior diameter (TA), thymus width (TW) and thymus height (TH) were measured. They were recorded as the longest measurements of the three axes of the fetal thymus, respectively. Finally, the surface area and volume of the thymus were obtained automatically. All the results for thymus glands in the present study were measured three times and the average was calculated. To check the reproducibility of the manual segmentation, the thymus was segmented manually twice simultaneously by two experienced pediatric radiologists to obtain an average value. The time interval between each round of manual segmentation was $\geq 1$ week.

Determination of thymus height and width and anteroposterior diameter. $\mathrm{TH}$, defined as the maximum distance between the base and apex of the thymus, was determined as was TW, defined as the maximum distance of the thymus along the horizontal plane and TA, defined as the longest distance perpendicular to the height and width of the thymus. The thymus was manually segmented twice simultaneously by two experienced anatomists to obtain an average value to check the reproducibility of manual segmentation.

Statistical analysis. The Student's t-test was employed, with GA as the confounding variable, to analyze the effect of gender on different measurements of the fetal thymus. The relationship between each measurement and GA was evaluated using regression analysis. The linear regression model was used and significant correlations were defined between data in presence of $R^{2}$ values of $>0.6$. Statistical analyses were performed using SPSS 17.0 software (SPSS Inc.). $\mathrm{P}<0.05$ was considered to indicate a statistically significant difference.

\section{Results}

Location and morphology of the fetal thymus. The thymus at 16 weeks' GA was clearly demonstrated. In the images of the FS-T2 TSE sequences, the thymus appeared heterogeneous with moderate signal intensity surrounded by many portions of thymus cortex with lower signal intensity (Fig. 2). The thymus was visualized more clearly in FS-T2 weighted MRI compared with other sequences. It is the major anterior component of the superior mediastinum lying immediately posterior to the manubrium of the sternum. The upper limit of the thymus reached the thyroid gland. The lower portion typically extended into the anterior mediastinum over the pericardial sac. The majority of the contour of axial images was roughly square, in which an asymmetric ' $\mathrm{X}$ ' configuration was evident in the coronal image. A signal of low intensity was observed at the edge of the thymus, while a high intensity signal was noted between the thymus and the lung. The interlobular septum was clearly shown, which was full of liquid and displayed a linear high signal. After 3D reconstruction, the shape of the thymus was successfully obtained (Fig. 3).

$T A, T W, T H, T S$ and volume (TV) of the thymus. The original measurements including TA, TW, TH, TS and TV of the thymus at a 16-39 week GA are given in Table I.

There were 33 male fetuses. The TA, TW, TH, TS and TV were $1.69 \pm 0.85 \mathrm{~cm}, 2.67 \pm 1.21 \mathrm{~cm}, 2.41 \pm 1.01 \mathrm{~cm}$, $17.04 \pm 13.83 \mathrm{~cm}^{2}$ and $3.31 \pm 3.52 \mathrm{~cm}^{3}$. There were 31 female fetuses. The TA, TW, TH, TS and TV were $1.66 \pm 0.77 \mathrm{~cm}$, 


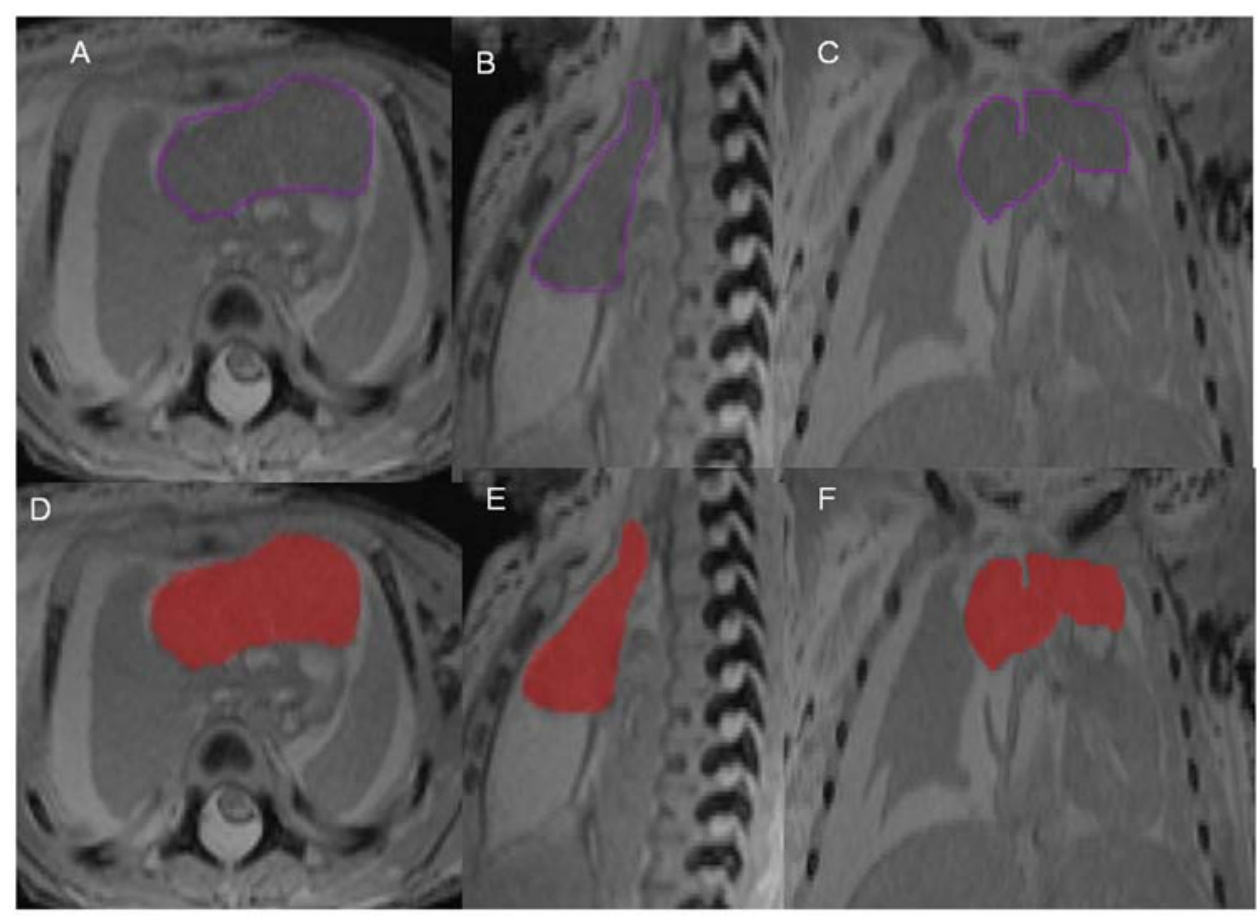

Figure 1. The 3D T2-weighted MRI images of the fetal thymus at 25 weeks GA using Amira 5.4 software. (A-C) The silhouette of the fetal thymus was artificial marked with purple lines on each image in the transverse, sagittal and coronal planes. (D-F) After tracing, the 3D software system automatically fills the selected area red for easy correction. 3D, three-dimensional; MRI, magnetic resonance imaging; GA, gestational age.
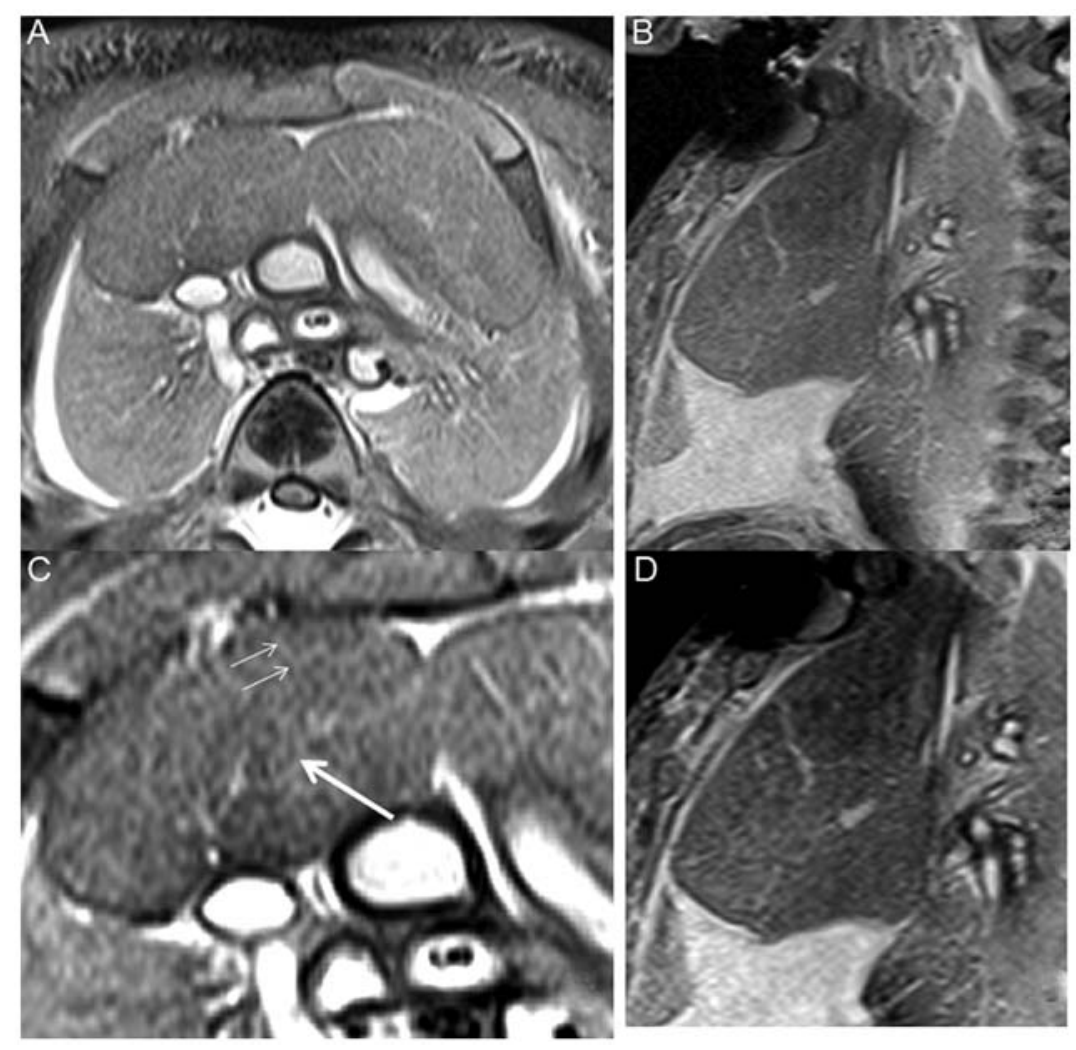

Figure 2. Transverse and sagittal sections of 25 week GA in FS-T2 TSE sequence. The shape of the thymus is irregular and mostly divided into two lobes. The axial contour appears roughly square and the sagittal appears cuneiform. (C and D) enlarged images of A and B, respectively. Heterogeneous moderate signal intensity (long arrow) were noticed in the thymus. Signals of lower intensity (two short arrows) were noticed in the surrounding tissues. GA, gestational age; TSE, turbo spin echo.

$2.37 \pm 0.99 \mathrm{~cm}, 2.24 \pm 0.91 \mathrm{~cm}, 13.84 \pm 11.79 \mathrm{~cm}^{2}$ and $2.94 \pm 3.69 \mathrm{~cm}^{3}$. No statistical differences were observed between the TA, TW, TH, TS and TV in the male and female fetuses $(\mathrm{P}>0.05$; Table II). 


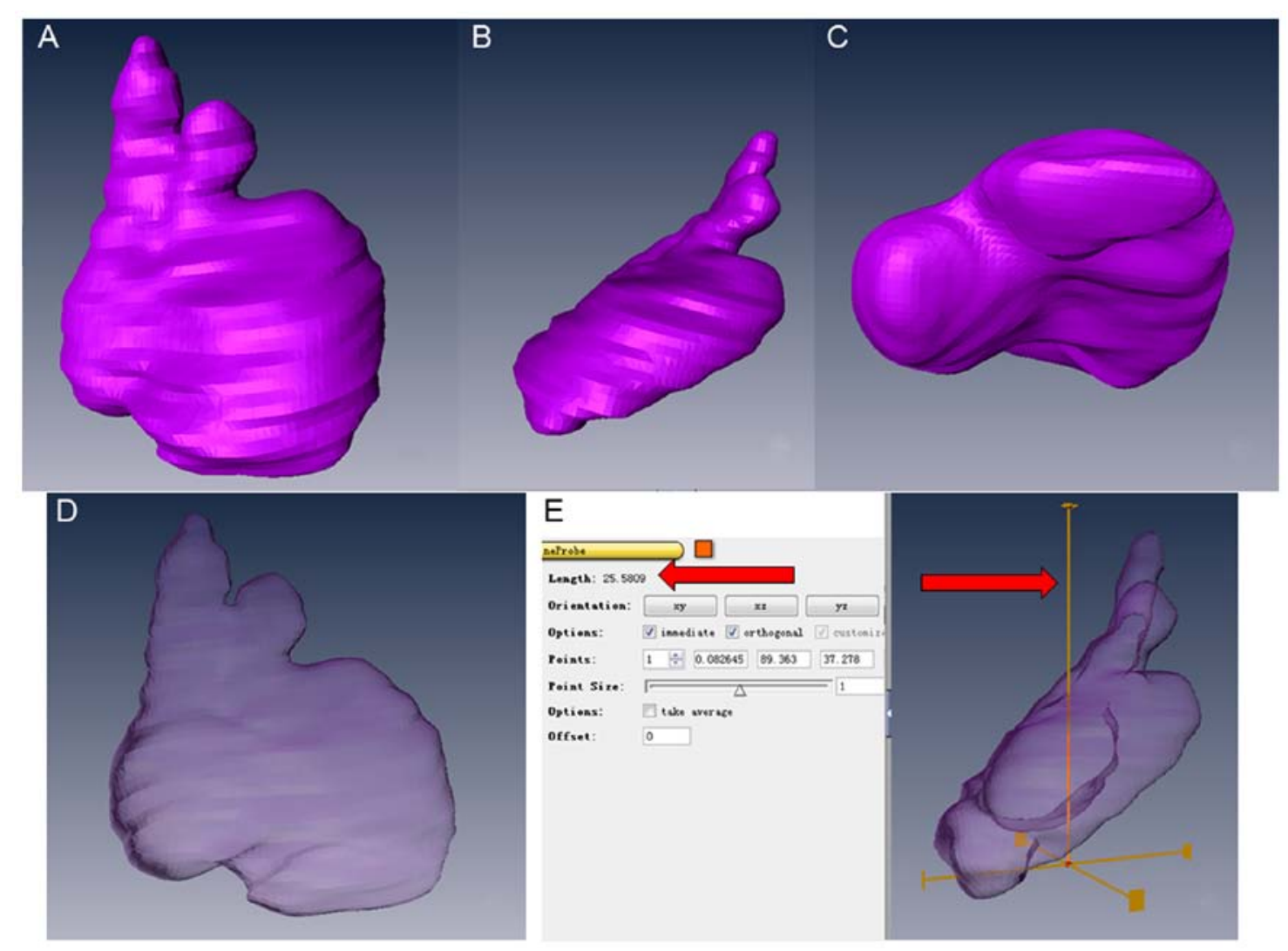

Figure 3. The 3D visualization model and linear measurements of the fetal thymus at 25 week GA. The 3D visualization model (A-C) of the fetal thymus of 25 weeks GA using Amira 5.4 for 3D data visualization, analysis and modeling. (D) Different display modes can be chosen according to preference. (E) TA, $\mathrm{TW}$ and TH are the longest length of the three axes of fetal thymus displayed automatically (red arrow). 3D, three-dimensional; GA, gestational age; TA, anteroposterior diameter; TH, thymus height; TW, thymus width.

The linear equations of the data and GA were as follows: $\mathrm{TA}=0.123 \times \mathrm{BA}-1.574 ; \mathrm{TW}=0.152 \times \mathrm{XA}-1.515$; $\mathrm{TH}=0.139 \times \mathrm{xA}-1.353 ; \mathrm{TS}=1.871 \times \mathrm{xA}-34.145 ;$ and $\mathrm{TV}=0.508 \times \mathrm{xA}-10.342$.

The slopes of the lines (Fig. 4) differed for different measurements. That for TS was the steepest (1.871), followed by that for TV (0.508), TW (0.152), TH (0.139) and TA (0.123). From the slope of the lines, it could be concluded that the measurements were developing at a different speed. The increase of thymus surface area and volume was the fastest, followed by its width. The TA increase was the slowest. However, linear regression analysis indicated the strongest correlation between TA and GA $\left(\mathrm{R}^{2}=0.844\right)$. Additionally, GA was correlated with TS $\left(\mathrm{R}^{2}=0.764\right)$, TH $\left(\mathrm{R}^{2}=0.753\right)$, TV $\left(\mathrm{R}^{2}=0.729\right)$ and $\mathrm{TW}\left(\mathrm{R}^{2}=0.675\right)$, respectively (Fig. 4$)$.

\section{Discussion}

The majority of the imaging studies on the fetal thymus have been focused on the prenatal ultrasonography, however, the structures of the intrauterine thymus could not be clearly displayed by US $(3,4,6,8,9)$. In a previous study, Leon-Luis et al (10) determined that the trans diameter and circumference of the fetal thymus (at 21-34 weeks GA) demonstrated no statistical differences among 17 cases using prenatal US and MRI. This study first reported the feasibility of MRI for the monitoring of fetal thymus. Nevertheless, the selected sequences could not effectively present the microanatomical structures of the thymus and the sample size was relatively small. Damodaram et al (21) utilized the T2 weighted single shot TSE sequence to compare the organ size and total fetal volume of the fetus $(n=20)$ with intrauterine growth retardation and those $(n=19)$ with normal development. This study revealed that turbo MRI contributed to the prenatal monitoring, but it lacked of real volume data for each organ. In addition, the microanatomical structures and normal ranges of the thymus were not mentioned.

Compared with in vivo diagnosis, post-mortem MRI (PMMRI) seems to be more reliable as it is free from the influence of maternal organs, pulse of arteries and movements of the fetus. Kang et al (11) proposed that 3T PMMRI was superior to post-mortem US for all anatomical regions except for the spine, particularly for fetuses of $\mathrm{GA} \geq 20$ weeks and the brain in fetuses $<20$ weeks. PMMRI remains the first line imaging investigation for perinatal autopsy. In the present study, the FS-T2 weighted TSE sequences could clearly display the morphological features and internal signals of the thymus. As previously described, medullary development was observed from a GA of 8 weeks and distinct cortical and medullary compartments by 16 weeks $(22,23)$. Histology of the thymus has shown that the periphery of the lobuli thymi is the cortex and the deep part is the medulla (24). However, the medulla is not completely surrounded by the cortex (25). Previous studies have shown abundant $\mathrm{T}$ cell progenitor cells in the cortex of the thymus $(8,17)$. By contrast, a small 
Table I. The original measurements of the fetal thymus $(n=64)$.

\begin{tabular}{|c|c|c|c|c|c|c|c|c|c|c|c|}
\hline GA & $\mathrm{TA}$ & TW & $\mathrm{TH}$ & $\mathrm{TS}$ & TV & GA & TA & TW & $\mathrm{TH}$ & $\mathrm{TS}$ & $\mathrm{TV}$ \\
\hline 16 & 0.49 & 0.85 & 0.79 & 1.39 & 0.11 & 25 & 1.68 & 2.45 & 2.49 & 15.48 & 3.10 \\
\hline 16 & 0.44 & 0.64 & 0.51 & 0.83 & 0.06 & 26 & 2.11 & 3.28 & 2.40 & 17.68 & 3.24 \\
\hline 17 & 0.72 & 0.72 & 1.01 & 2.80 & 0.16 & 26 & 2.23 & 3.64 & 2.77 & 23.32 & 3.59 \\
\hline 17 & 0.39 & 0.90 & 0.67 & 0.94 & 0.05 & 27 & 1.71 & 1.81 & 2.25 & 10.56 & 1.70 \\
\hline 19 & 0.58 & 1.03 & 1.28 & 2.97 & 0.28 & 27 & 1.68 & 3.34 & 3.00 & 20.96 & 3.13 \\
\hline 19 & 0.77 & 1.06 & 1.56 & 3.30 & 0.34 & 28 & 2.14 & 3.61 & 2.92 & 21.06 & 4.22 \\
\hline 20 & 0.56 & 1.41 & 1.40 & 3.13 & 0.21 & 28 & 1.50 & 2.20 & 2.59 & 13.21 & 3.06 \\
\hline 20 & 0.84 & 1.69 & 1.06 & 3.80 & 0.35 & 28 & 1.97 & 2.26 & 2.56 & 12.12 & 2.08 \\
\hline 20 & 0.76 & 2.18 & 1.55 & 5.45 & 0.51 & 28 & 1.40 & 2.15 & 0.99 & 6.07 & 0.64 \\
\hline 20 & 0.68 & 1.19 & 1.09 & 2.28 & 0.21 & 28 & 2.37 & 3.39 & 2.48 & 19.74 & 4.32 \\
\hline 21 & 1.40 & 1.76 & 1.00 & 4.88 & 0.48 & 28 & 1.65 & 2.82 & 3.10 & 15.29 & 2.57 \\
\hline 21 & 1.10 & 1.21 & 1.74 & 4.85 & 0.56 & 28 & 1.86 & 2.52 & 3.04 & 16.65 & 2.69 \\
\hline 22 & 0.87 & 1.82 & 1.82 & 7.80 & 1.05 & 29 & 1.78 & 1.36 & 2.12 & 7.75 & 1.29 \\
\hline 22 & 0.72 & 2.28 & 0.75 & 7.81 & 0.92 & 29 & 2.63 & 4.14 & 3.02 & 32.00 & 6.60 \\
\hline 22 & 1.14 & 1.69 & 1.59 & 4.43 & 0.34 & 29 & 2.20 & 2.05 & 2.43 & 14.13 & 2.29 \\
\hline 22 & 1.12 & 1.58 & 2.17 & 6.37 & 0.62 & 30 & 2.18 & 3.37 & 3.37 & 31.60 & 8.43 \\
\hline 22 & 1.00 & 2.14 & 2.28 & 7.69 & 1.04 & 30 & 2.25 & 3.95 & 3.53 & 33.38 & 6.57 \\
\hline 22 & 1.10 & 2.03 & 1.63 & 7.55 & 1.25 & 30 & 1.56 & 2.11 & 2.65 & 9.36 & 0.92 \\
\hline 23 & 1.19 & 1.66 & 1.50 & 5.02 & 0.60 & 31 & 2.10 & 3.31 & 2.47 & 18.50 & 3.88 \\
\hline 23 & 1.41 & 1.95 & 1.82 & 7.07 & 0.85 & 32 & 2.13 & 3.75 & 2.50 & 27.51 & 4.35 \\
\hline 23 & 1.18 & 1.97 & 1.56 & 6.35 & 0.56 & 34 & 2.12 & 2.38 & 2.70 & 14.03 & 2.37 \\
\hline 24 & 1.02 & 2.12 & 1.85 & 8.20 & 0.82 & 34 & 2.11 & 2.88 & 3.74 & 22.46 & 5.83 \\
\hline 24 & 1.54 & 2.43 & 1.83 & 9.98 & 1.08 & 34 & 2.71 & 5.07 & 4.02 & 39.88 & 9.06 \\
\hline 24 & 1.76 & 3.12 & 1.88 & 12.18 & 1.80 & 35 & 2.87 & 4.62 & 2.79 & 36.40 & 9.90 \\
\hline 24 & 1.88 & 2.46 & 2.33 & 12.86 & 1.94 & 35 & 3.27 & 3.04 & 2.34 & 22.17 & 4.23 \\
\hline 24 & 1.66 & 2.42 & 2.60 & 11.30 & 1.61 & 36 & 2.52 & 3.88 & 3.76 & 26.70 & 4.80 \\
\hline 24 & 1.19 & 1.54 & 2.15 & 6.67 & 0.93 & 36 & 2.91 & 4.93 & 4.62 & 45.31 & 11.90 \\
\hline 24 & 0.67 & 1.44 & 1.64 & 3.63 & 0.31 & 38 & 2.74 & 4.14 & 3.79 & 37.14 & 12.21 \\
\hline 25 & 1.42 & 2.36 & 2.14 & 9.16 & 1.08 & 38 & 2.67 & 3.06 & 3.55 & 31.77 & 8.37 \\
\hline 25 & 1.45 & 2.47 & 2.40 & 11.23 & 1.69 & 38 & 3.25 & 4.58 & 4.52 & 52.07 & 12.04 \\
\hline 25 & 1.01 & 1.56 & 2.39 & 7.45 & 0.94 & 39 & 3.23 & 4.32 & 3.70 & 48.55 & 12.80 \\
\hline 25 & 2.00 & 3.32 & 2.86 & 17.29 & 2.93 & 39 & 3.83 & 4.48 & 3.96 & 42.08 & 12.79 \\
\hline
\end{tabular}

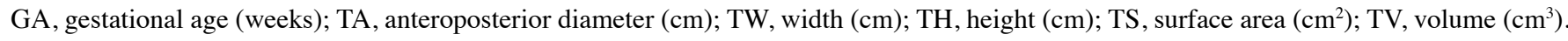

Table II. The measurements of thymus between sex groups.

\begin{tabular}{|c|c|c|c|c|c|c|}
\hline Groups & $\mathrm{N}$ & $\mathrm{TA}$ & TW & $\mathrm{TH}$ & TS & TV \\
\hline Male & 33 & $1.69 \pm 0.85$ & $2.67 \pm 1.21$ & $2.41 \pm 1.01$ & $17.04 \pm 13.83$ & $3.31 \pm 3.52$ \\
\hline Female & 31 & $1.66 \pm 0.77$ & $2.37 \pm 0.99$ & $2.24 \pm 0.91$ & $13.84 \pm 11.79$ & $2.94 \pm 3.69$ \\
\hline t-value & & 0.164 & 1.068 & 0.742 & 0.994 & 0.413 \\
\hline P-value & & 0.121 & 0.079 & 0.642 & 0.132 & 0.799 \\
\hline
\end{tabular}

GA, gestational age (weeks); N, number; TA, anteroposterior diameter $(\mathrm{cm})$; TW, width $(\mathrm{cm})$; TH, height $(\mathrm{cm})$; TS, surface area $\left(\mathrm{cm}^{2}\right)$; $\mathrm{TV}$, volume $\left(\mathrm{cm}^{3}\right)$.

population of $\mathrm{T}$ cell progenitor cells were noticed in the medulla. In addition, other cells types were noticed in the medulla, such as thymic epithelial cells, neural crest-derived mesenchymal cells, endothelial cells and dendritic cells (11). This may help to explain why the cortex and medulla can be clearly distinguished on MRI. 
A
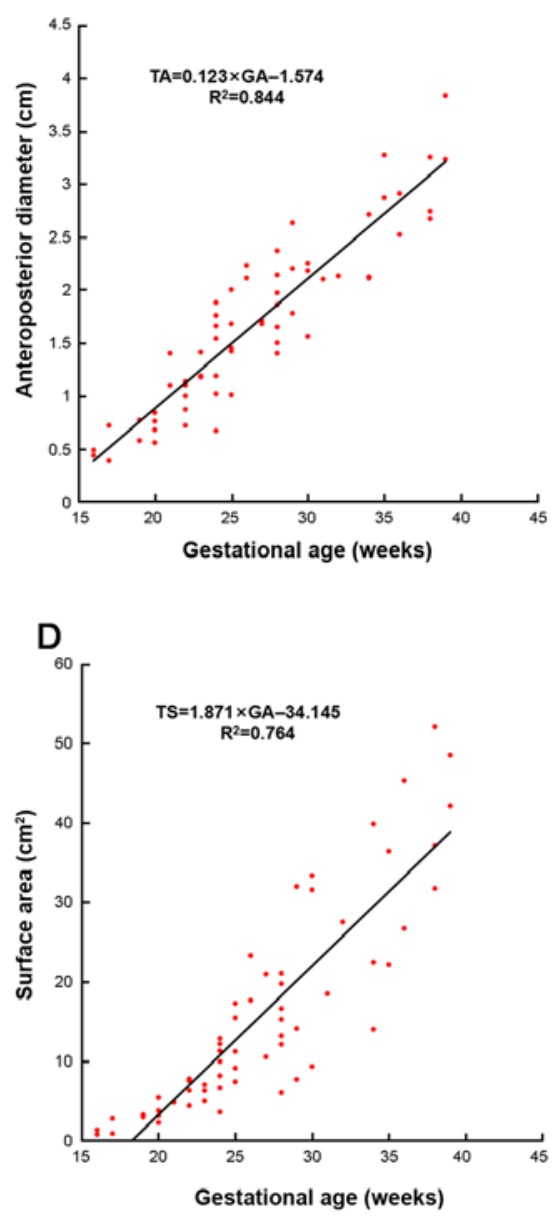

B
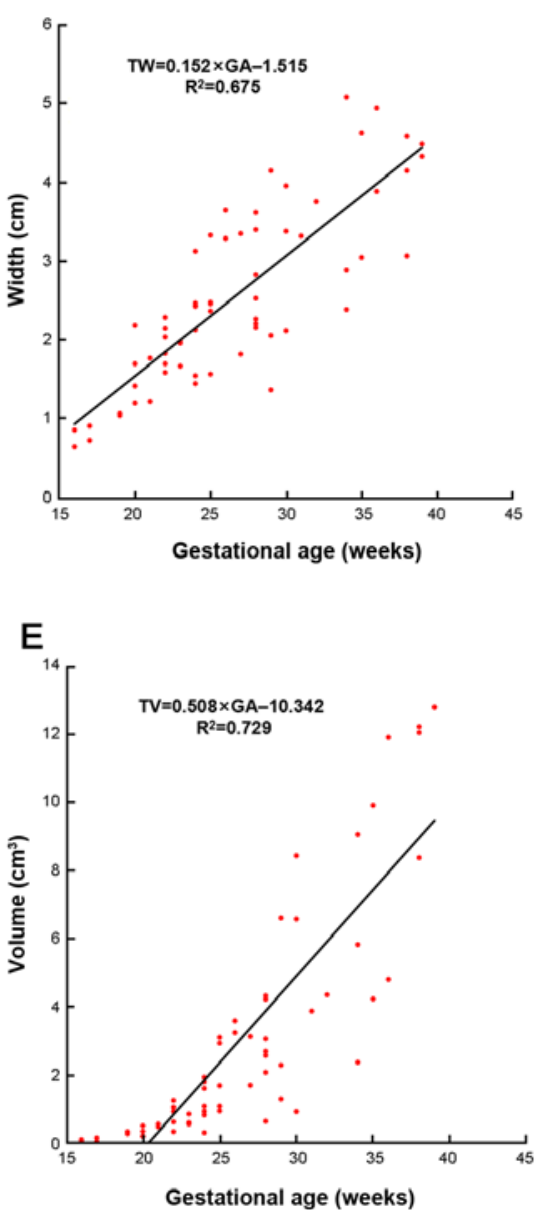

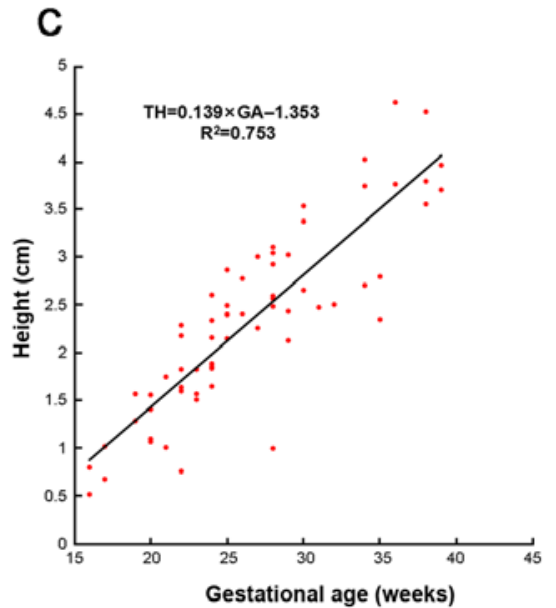

Figure 4. The statistical results between all the measurements and GA. The scattergrams, best-fit equations and correlation coefficients (R2) of (A) TA, (B) TW, (C) TH, (D) TS, (E) TV and GA. All the measurements linearly increase with GA. Each symbol represents a single fetus. GA, gestational age; TA, anteroposterior diameter; TW, thymus width; TH, thymus height; TS, thymus.

It has been previously indicated that thymic size is influenced by a series of hormones, including sex steroids and those involve in the hypothalamic-pituitary-adrenal axes (26). It has been revealed that sex hormones receptors are expressed in both thymocytes and TECs $(26,27)$. Castration of male rodents results in significant enlargement of thymus $(27,28)$. Nevertheless, the data from the present study demonstrated that there were no statistical differences between the gender and thymic size, which was in line with a previous study (6) in which sonography was used to measure the transverse diameter and perimeter of 59 normal thymuses and demonstrated no effects of fetal gender on the thymus.

In prenatal MR imaging, in addition to morphological subjective evaluation of structures, quantitative information may be important in the diagnosis of anomalies. Graham et al (13) suggested that the thymus was morphologically complete at 16-20 gestational weeks upon maturation. Li et al (29) proposed that the volume of the thymus was more appropriate to evaluate the size of the thymus. Cho et al (30) reported that it is difficult to confirm the size and arrangement of the large blood vessels by analyzing the anteroposterior diameter. Unlike previous studies $(29,30)$, the present study demonstrated that the anteroposterior diameter of the thymus was more closely associated with GA than other factors $\left(\mathrm{R}^{2}=0.844\right)$. Additionally, the majority of the measured values were lower than the previous data obtained from 3D US (29). It is hypothesized that the data from the present study can provide more accurate information about the thymus because they are based on the high resolution of post-mortem 3.0T MRI.

The growth curve generated in the present study can be considered as a model forclinical applications. Somestudies have indicated that 2D data obtained from US are less efficient than 3D data in assessing the morphometry of the thymus $(14,20,31)$. For this reason, 2D technique could only reflect the size of the thymus in a single dimension or plane, but 3D volume measurement is more objective and reliable in reflecting the thymic size. The present study, based on MRI, demonstrated that parameters measured in $2 \mathrm{D}$ were as closely correlated with GA as that of 3D data.

The present study reported a new and reliable method for the study of fetal thymus development and maturation combining the image of thymus obtained by post-mortem 3.0T MRI scanning with powerful 3D software. More intuitive and accurate morphological data of thymus were obtained. The 3D reconstruction analysis is superior to traditional pathological analysis in the following aspects: First, 3D reconstruction analysis displayed the fetal thymus surface and inner structures without excising the thymus, which precluded the 
possibility of deformation by gravity during excision. Second, arbitrary slices can be obtained by postprocessing on ordinary computers. Third, accurate measurements of each component can be easily and precisely obtained with the thymus in its natural state. Therefore, Amira 5.4 software may be useful for the research of irregular organs. It is hoped that it will be applied to the monitoring of fetal thymus in uterus in the future.

There are some limitations to the present study. First, the range of GA was large, but the sample size of 64 fetuses was small. To be exact, only two specimens at a GA of 31 weeks $(n=1)$ and 32 weeks $(n=1)$ were included. No specimens at a GA of 18, 33 and 37 weeks were included, which may lead to bias to the conclusion of the present study. Second, an undetected anomaly may present in the selected specimens.

The present study is the first to use PMMRI and Amira 5.4 software to measure the normal fetal thymus. PMMRI may play a vital role in providing more anatomical detail than US and in vivo fetal MRI. The results of the present study may serve as a valuable reference for fetal MRI applied in vivo in prenatal diagnosis.

\section{Acknowledgements}

Not applicable.

\section{Funding}

The present study was supported by the National Natural Science Foundation of China (grant no. 31371213) and the National Natural Science Foundation of Shandong Province (grant no. ZR2013HM091).

\section{Availability of data and materials}

The datasets used and/or analyzed during the current study are available from the corresponding author on reasonable request.

\section{Authors' contributions}

LY and XL conceived and designed the experiments. LY, JC, ZW, JD and HX performed the experiments. LY, LZ and XW analyzed the data. LY and LZ contributed reagents/materials/analysis tools. LY and XL wrote the manuscript. YW and JC performed the collection and storage of fetuses. All authors read and approved the final manuscript.

\section{Ethics approval and consent to participate}

The present study was carried out with approval of the Ethical Committee of School of Medicine, Shandong University (approval no. 2012033). Written informed consent was obtained from the guardian(s) of each patient.

\section{Patient consent for publication}

Not applicable.

\section{Competing interests}

The authors declare that they have no competing interests.

\section{References}

1. Ferguson AC: Prolonged impairment of cellular immunity in children with intrauterine growth retardation. J Pediatr 93: 52-56, 1978.

2. McDade TW, Beck MA, Kuzawa CW and Adair LS: Prenatal undernutrition and postnatal growth are associated with adolescent thymic function. J Nutr 131: 1225-1231, 2001.

3. Felker RE, Cartier MS, Emerson DS and Brown DL: Ultrasound of the fetal thymus. J Ultrasound Med 8: 669-673, 1989.

4. Gamez F, De Leon-Luis J, Pintado P, Perez R, Robinson JN, Antolin E, Ortiz-Quintana L and Santolaya-Forgas J: Fetal thymus size in uncomplicated twin and singleton pregnancies. Ultrasound Obstet Gynecol 36: 302-307, 2010.

5. Lamouroux A, Mousty E, Prodhomm O, Bigi N, Le Gac MP, Letouzey V, De Tayrac R and Mares P: Absent or hypoplastic thymus: A marker for 22q11.2 microdeletion syndrome in case of polyhydramnios. J Gynecol Obstet Biol Reprod (Paris) 45: 388-396, 2016.

6. De Leon-Luis J, Gamez F, Pintado P, Antolin E, Pérez R, Ortiz-Quintana L and Santolaya-Forgas J: Sonographic measurements of the thymus in male and female fetuses. J Ultrasound Med 28: 43-48, 2009.

7. Warncke K, Lickert R, Eitel S, Gloning KP, Bonifacio E, Sedlmeier EM, Becker P, Knoop J, Beyerlein A and Ziegler AG: Thymus growth and fetal immune responses in diabetic pregnancies. Horm Metab Res 49: 892-898 2017.

8. Yinon Y, Zalel Y, Weisz B, Mazaki-Tovi S, Sivan E, Schiff E and Achiron R: Fetal thymus size as a predictor of chorioamnionitis in women with preterm premature rupture of membranes. Ultrasound Obstet Gynecol 29: 639-643, 2007.

9. Zalel Y, Gamzu R, Mashiach S and Achiron R: The development of the fetal thymus: An in utero sonographic evaluation. Prenat Diagn 22: 114-117, 2002.

10. De Leon-Luis J, Ruiz Y, Gamez F, Pintado P, Oyelese Y, Pereda A, Ortiz-Quintana L and Santolaya-Forgas J: Comparison of measurements of the transverse diameter and perimeter of the fetal thymus obtained by magnetic resonance and ultrasound imaging. J Magn Reson Imaging 33: 1100-1105, 2011.

11. Kang X, Shelmerdine SC, Hurtado I, Bevilacqua E, Hutchinson C, Mandalia U, Segers V, Cos Sanchez T, Cannie MM, Carlin A, et al: Postmortem fetal imaging: A prospective blinded comparison study of 2-dimensional ultrasound with MR imaging. Ultrasound Obstet Gynecol 53: 229-238, 2019.

12. Gordon J and Manley NR: Mechanisms of thymus organogenesis and morphogenesis. Development 138: 3865-3878, 2011.

13. Graham A, Okabe M and Quinlan R: The role of the endoderm in the development and evolution of the pharyngeal arches. J Anat 207: 479-487, 2005.

14. Guihard-Costa AM, Menez F and Delezoide AL: Organ weights in human fetuses after formalin fixation: Standards by gestational age and body weight. Pediatr Dev Pathol 5: 559-578, 2002.

15. Liu F, Zhang Z, Lin X, Teng G, Meng H, Yu T, Fang F, Zang F, $\mathrm{Li} Z$ and Liu S: Development of the human fetal cerebellum in the second trimester: A post mortem magnetic resonance imaging evaluation. J Anat 219: 582-588, 2011.

16. Zhang Z, Liu S, Lin X, Teng G, Yu T, Fang F and Zang F: Development of fetal brain of 20 weeks gestational age: Assessment with post-mortem magnetic resonance imaging. Eur J Radiol 80: e432-e439, 2011.

17. Di Naro E, Cromi A, Ghezzi F, Raio L, Uccella S, D'Addario V and Loverro G: Fetal thymic involution: A sonographic marker of the fetal inflammatory response syndrome. Am J Obstet Gynecol 194: 153-159, 2006.

18. Meng H, Zhang Z, Geng H, Lin X, Feng L, Teng G, Fang F, Zang $\mathrm{F}$ and Liu S: Development of the subcortical brain structures in the second trimester: Assessment with 7.0-T MRI. Neuroradiology 54: 1153-1159, 2012.

19. Zhang Z, Hou Z, Lin X, Teng G, Meng H, Zang F, Fang F and Liu S: Development of the fetal cerebral cortex in the second trimester: Assessment with 7T postmortem MR imaging. AJNR Am J Neuroradiol 34: 1462-1467, 2013.

20. Jelev L and Surchev L: Radial artery coursing behind the biceps brachii tendon: Significance for the transradial catheterization and a clinically oriented classification of the radial artery variations. Cardiovasc Intervent Radiol 31: 1008-1012, 2008.

21. Damodaram MS, Story L, Eixarch E, Patkee P, Patel A, Kumar S and Rutherford M: Foetal volumetry using magnetic resonance imaging in intrauterine growth restriction. Early Hum Dev 88 (Suppl 1): S35-S40, 2012. 
22. Chung L, Maestas DR Jr, Housseau F and Elisseeff JH: Key players in the immune response to biomaterial scaffolds for regenerative medicine. Adv Drug Deliv Rev 114: 184-192, 2017.

23. Gordon J, Wilson VA, Blair NF, Sheridan J, Farley A, Wilson L, Manley NR and Blackburn CC: Functional evidence for a single endodermal origin for the thymic epithelium. Nat Immunol 5: 546-553, 2004

24. Farley AM, Morris LX, Vroegindeweij E, Depreter ML, Vaidya H, Stenhouse FH, Tomlinson SR, Anderson RA, Cupedo T, Cornelissen JJ and Blackburn CC: Dynamics of thymus organogenesis and colonization in early human development. Development 140: 2015-2026, 2013.

25. Von Gaudecker B: Functional histology of the human thymus. Anat Embryol (Berf) 183: 1-15, 1991.

26. Hince M, Sakkal S, Vlahos K, Dudakov J, Boyd R and Chidgey A The role of sex steroids and gonadectomy in the control of thymic involution. Cell Immunol 252: 122-138, 2008.

27. Olsen NJ, Olson G, Viselli SM, Gu X and Kovacs WJ: Androgen receptors in thymic epithelium modulate thymus size and thymocyte development. Endocrinology 142: 1278-1283, 2001.
28. Williams KM, Lucas PJ, Bare CV, Wang J, Chu YW, Tayler E, Kapoor V and Gress RE: CCL25 increases thymopoiesis after androgen withdrawal. Blood 112: 3255-3263. 2008.

29. Li L, Bahtiyar MO, Buhimschi CS, Zou L, Zhou QC and Copel JA: Assessment of the fetal thymus by two- and three-dimensional ultrasound during normal human gestation and in fetuses with congenital heart defects. Ultrasound Obstet Gynecol 37: 404-409, 2011.

30. Cho JY, Min JY, Lee YH, McCrindle B, Hornberger LK and Yoo SJ: Diameter of the normal fetal thymus on ultrasound. Ultrasound Obstet Gynecol 29: 634-638, 2007.

31. El-Haieg DO, Zidan AA and El-Nemr MM: The relationship between sonographic fetal thymus size and the components of the systemic fetal inflammatory response syndrome in women with preterm prelabour rupture of membranes. BJOG 115: 836-841, 2008.

This work is licensed under a Creative Commons Attribution-NonCommercial-NoDerivatives 4.0 International (CC BY-NC-ND 4.0) License. 